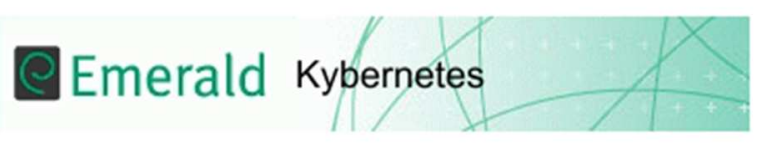

\title{
An Optimization Model of the Acceptable Consensus and Its Economic Significance
}

\begin{tabular}{|r|l|}
\hline Journal: & Kybernetes \\
\hline Manuscript ID: & K-04-2014-0070.R1 \\
\hline Manuscript Type: & Research Paper \\
\hline Keywords: & $\begin{array}{l}\text { Group decision making, consensus, collectively acceptable consensus, } \\
\text { information entropy }\end{array}$ \\
\hline \multicolumn{2}{|l}{} \\
\hline
\end{tabular}

\section{SCHOLARONE $^{\text {"x }}$ \\ Manuscripts}




\title{
An Optimization Model of the Acceptable Consensus and Its Economic Significance
}

\begin{abstract}
Purpose-This paper aims to construct an optimal resource reallocation model of the limited resource by a moderator for reaching the greatest consensus, and shows how to reallocate the limited resources by using optimization methodology once the consensus opinion is reached. Moreover, this paper also devotes to theoretically exploring when or what is the condition that the group decision making (GDM) system is stable; and when new opinions enter into the GDM, how the level of consensus changes.

Design/methodology/approach- By minimizing the differences between the individuals' opinions and the collective consensus opinion, this paper constructs a consensus optimization model and shows that the objective weights of the individuals are actually the optimal solution to this model.

Findings- If all individual deviations of the DMs from the consensus balance each other out, the information entropy theorem shows this GDM is most stable, and economically each individual DM gets the same optimal unit of compensation. Once the consensus opinion is determined and each individual opinion of the DMs is under an acceptable consensus level, the consensus is still acceptable even if additional DMs are added, and the moderator's cost is still no more than a fixed upper limitation.

Originality/value- The optimization model based on acceptable consensus is constructed in this paper, and its economic significance, including the theoretical and practical significance, is emphatically analyzed: it is shown that the weight information of the optimization model carries important economic significance. Besides, some properties of the proposed model are discussed by analyzing its particular solutions: the stability of the consensus system is explored by introducing information entropy theory and variance distribution; in addition, the effect of adding new DMs on the stability of the acceptable consensus system is discussed by analyzing the convergence of consensus level: it is also built up the condition that once the consensus opinion is determined, the consensus degree will not decrease even when additional DMs are added to the GDM.
\end{abstract}

Key Words Group decision making, consensus, collectively acceptable consensus, information entropy

Paper type Research paper

\section{Introduction}

The method of group decision making (GDM) represents an effective means to deal with the systemic problem of group complexity that concerns with expert decision making and negotiation, 
such as situations in sport competitions, program reviews, parliamentary elections, and various international negotiations. In the process of a GDM (Arrow, 1963), there are various kinds of conflicts and divergences among the individual Decision Makers (DMs), interest groups, individuals, and interest groups. After many rounds of debate over different opinions, exchanges of ideas, negotiations on different positions, and making compromises, the DMs may reach a consensus in the end (GonzalezPachon and Romero, 2011). This consensus decision making is also a process of reaching an ultimate agreement. It means that the consensus also stands for a method through which the entire group of the DMs eventually comes to a settlement. Here, by consensus, it is defined as "an opinion or position reached by a group as a whole" according to the American Heritage Dictionary. Another definition reads that consensus (Ben-Arieh and Easton, 2009; Ness and Hoffman, 1998) represents "a decision that has been reached when most members of the team agree on a clear option and the few who oppose it think they have had a reasonable opportunity to influence that choice; all team members agree to support the decision". In this sense, "all consensus reaching processes proceed in a multistage setting, i.e., the individual DMs change their opinions step by step until, possibly, some consensus is reached (Herrera-Viedma et al., 2014 )". Through consensus, we not only work to arrive at a better solution, but also promote cohesion, trust, and harmony of the community involved.

From the point of view of mathematical optimization, the process of reaching consensus (or consensus process) involves maximizing the agreement among a group of DMs. Generally, there are two kinds of methodologies towards reaching consensus. One is to use the objective weights obtained through using optimization models that minimize the weighted sum of the dissimilarities between the DMs, or minimize distance between the individuals' opinions and the collective opinion, and then combine the individuals' opinions by utilizing aggregation operators. The characteristics of this methodology are that the DMs in the GDM do not need to modify their opinions to converge to the ultimate collective opinion. For example, Lee (2002) developed an optimal consensus method for GDM environment by minimizing the sum of weighted dissimilarity among the aggregated consensus and the individuals' opinions. Wang and Parkan (2006) proposed the least squares distance method and the defuzzification-based least squares method for the assessment of the weights to be associated with fuzzy opinions. Chen and Lee (2012) presented an autocratic consensus decision making using group recommendations based on the interval linguistic labels ordered weighted average (ILLOWA) operator and likelihood-based comparison relations. Ben-Arieh and Chen (2006) presented a procedure for handling an autocratic GDM process under linguistic assessments by introducing a new linguisticlabels aggregation operation. Xu and Cai (2011) developed a number of goal programming models and quadratic programming models based on the idea of maximizing consensus to derive the importance 
weights of fuzzy preference relations and multiplicative preference relations, and then derived iterative algorithms for reaching acceptable levels of consensus for GDM.

Another approach of GDM represents a dynamic and iterative process of modifying opinions in order to reach an ultimate consensus. Some methodologies along this line focus on how to modify individual DMs' opinions in order to increase the level of consensus. For example, Bryson (1996) proposed a framework for using consensus relevant information embedded in the preference data to assess the current level of group consensus, and to support the process of consensus building in 1996. And in 2007, Ben-Arieh and Easton (2007) introduced the concept of minimum cost consensus, constructed a multi-criteria consensus model under linear cost opinion elasticity, and presented lineartime algorithms to find the minimum cost consensus. Then, Ben-Arieh et al. (2009) presented new algorithms to find the minimum cost consensus for three different models: the model of consensus at the minimum quadratic cost, the model of $\varepsilon$ consensus at the minimum quadratic cost, and the model of budget-constrained consensus at the minimum quadratic cost. Later, Zhang et al. (2011) generalize the works of Ben-Arieh et al. They proposed novel models to achieve the minimum-cost consensus under different aggregation operators, and developed a linear-programming methodology to solve the models. While others focus on group consensus for the dynamics of the discrete-time multi-agent system. For examples, Miao and Ma (2015) proposed consensus protocols for discretetime and continuous-time multi-agent systems to investigates consensus for the first-order multi-agent systems with nonlinear input constraints; Yang et al. (2014) used the Lyapunov function to derive the consensus conditions of discrete-time multi-agent systems; And Feng et al. (2014) discuss consensus problems for the second-order multi-agent systems.

Being different from these two kinds of consensus methodologies, this paper focuses on constructing a novel optimization consensus model, and discusses the particular meaning of objective weights of DMs. According to the viewpoint of Bryson (1996, 1997), unless there is an acceptable level of consensus, it is premature to use mathematical models to generate the objective weights (preference vector). Therefore, in our new consensus model, we first suppose that the current level of consensus in GDM is acceptable. In such a context, (1), a consensus opinion or an acceptable consensus opinion is also supposed to exist, and a moderator introduced in GDM is entrusted that he/she can persuade each individual DM to change his/her opinion towards the consensus opinion by paying the cost (consuming resources such as time, money) to the individuals, and the individuals' opinions that have been modified many times are within a threshold (acceptable) value of deviation of this consensus opinion. (2), the weight of each DM is objectively obtained through an optimization consensus model that is constructed by minimizing the aggregation of the deviations between the individuals' opinions and 
consensus opinion. Economically, it will also be shown that each weight actually represents an optimal unit of compensation on the individual DM for changing his/her opinion towards the consensus opinion. And (3), the convergence of consensus level will be analyzed. In other words, the effect of adding new DMs on the stability of the acceptable consensus system will be explored in this paper.

This paper is structured as follows. Section 2 provides the background information and introduces the hypothesis of consensus decision making. Section 3 contains three parts. The first part describes the principle of constructing the optimization consensus model based on optimal resource reallocation, and explores properties of this model in order to produce a more generalized optimization consensus model on the basis of ordered weighted aggregation (OWA) operator. The second part establishes the conditions for the existence of general solutions of the consensus model. And the last part discusses the special properties of the acceptable consensus model when new DMs enter into the GDM. Section 4 and 5 use numerical examples and a example of demolition and relocation of an urban building to illustrate how the proposed models work. Section 6 concludes the paper.

\section{Background and Basic Hypothesis}

In the process of consensus decision making, DMs are expected to participate equally by contributing opinions (or suggestions). A minimum number of individual DMs is necessary to provide an accurate representation of the decision-making problem and to take into account of all perspectives in the group so that the eventual decision outcome is in everyone's interests, and meets the actual circumstances better. Consensus decision making involves three basic steps: the process of opinion assimilation (information fusion) (Herrera et al., 2005), opinion optimization, and opinion aggregation. The consensus process (Ben-Arieh and Easton, 2007) is also a dynamic and interactive group decision process, so a moderator (Ben-Arieh and Easton, 2009), responsible for the whole process of consensus decision making and for coordinating each of the individual DMs to gradually change his or her opinions towards a collective opinion, is supposed to exist. He/she is required to represent the collective interest and to help reach the consensus, and has been predetermined and possesses an effective leadership and strong interpersonal communication and negotiation skills (Herrera-Viedma et al., 2014; Cabrerizo et al., 2010; Herrera et al., 1996; Pérez et al., 2013). When the moderator tries to persuade individual DMs to change their opinions in order to reach a consensus, he/she is supposed to mobilize all possible forms of resources or pay a cost (Ben-Arieh and Easton, 2007, 2009; Zhang et al., 2011), be they material, financial, human, and informational. When the individuals have to change their opinions towards a collective opinion, they deserve to be compensated or to be rewarded (Gong et al., 2015a, Gong et al., 2015b). 


\subsection{Opinion Assimilation}

In GDM, various opinions are expressed by the DMs representing their varied interests, hobbies, and knowledge structures. Each expressed opinion, presenting different structures, includes a utility function (Houthakker, 1950), a judgment matrix (Xu, 2005, 2007; Ramanathan R and U, 2010; Bryson and Joseph, 1999; Chiclana et al., 2002; Herrera-Viedma et al., 2005, 2007, 2007; Saaty and Rogers, 1976; Umano et al., 1998) (or preference relations, such as multiplicative preference relation, additive preference relation, interval preference relation, natural linguistic preference relation, and grey preference relation), attribute values, etc. It reflects a certain degree of confidence regarding the decision making problem. There also exist differences in the scales of criteria even when the opinions are the same. For example, suppose that we invite two DMs to review a scientific research paper. They naturally have different sets of evaluation criteria regarding the meaning of "excellent". Suppose that the first DM holds the view that an excellent paper scores at least 85, while the other DM thinks an excellent paper scores at least 90. Consequently, the moderator tries to construct an appropriate mapping transformation (function) for the purpose of establishing decision rules in order to unify or assimilate these structures of different kinds of opinions and scales of criteria. We call this process opinion (information) assimilation. The following diagram shows the step of opinion assimilation by the moderator:

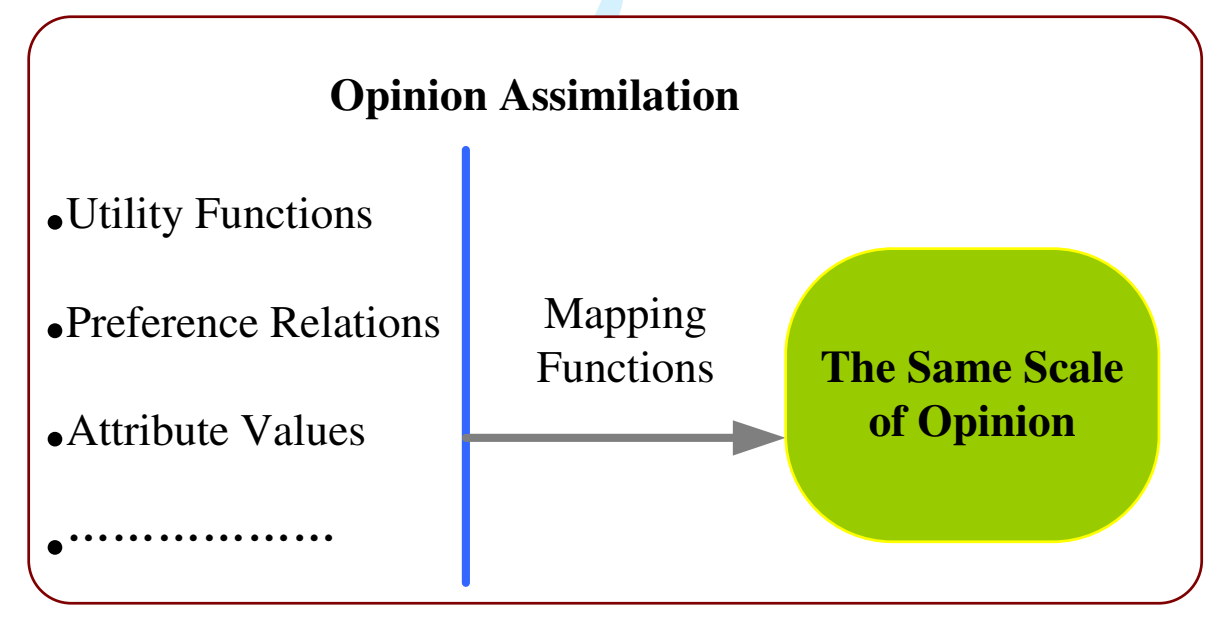

Figure 1: Step 1 of the Moderator: Opinion Assimilation

\subsection{Opinion Optimization}

The process of opinion optimization is essentially the minimization of the differences among the individuals' opinions or of the divergences between the individuals' opinions and collective opinion. Opinion optimization is achieved in two ways: One is to fully consider the opinion differences between 
the individuals, and then to minimize the individuals' differences (Wang and Parkan, 2006; Xu and Cai, 2011); the other is to fully consider the opinions between the individuals and the group, and then minimize the deviation between the individuals' opinions and the collective consensus opinion (Ben-Arieh and Easton, 2007, 2009; Zhang et al., 2011). In the process of consensus decision making, it is hard to obtain a completely consensus opinion that satisfies the interest of every individual. Usually, there always exists a difference between an individual DM's opinion and the collective opinion for such reasons as that: (1) each individual opinion contains limitation. That is, there always are bounded rationality (Simon, 1991), prejudice or bias, and preference in each individual opinion; and (2) there are complexity and conflicts in the collective opinion. That is, it is hard to obtain a completely identical opinion, even though all the individuals have similar values, backgrounds, abilities, knowledge structures, experiences, etc., in reality. For the sake of achieving consensus, on one hand, the moderator in GDM trusts that he can persuade each individual to change his (her) opinion to an ideal (consensus) value by paying the cost (consuming resources such as time, money). On the other hand, all individual DMs may expect to receive returns for changing their opinions towards the ideal opinion. In other words, in the process of consensus reaching, the moderator expects to pay his fees to obtain consensus, and each individual DM hopes to receive his/her share of compensation because he/she has made sacrifices in order to satisfy the collective interest. Under such circumstances, the group consensus opinion is often assumed to exist due to the existence of the moderator. During the process, because the moderator, who represents the group interest, tries to persuade each DM to alter his/her opinion, the individual DMs' opinions and the group consensus in fact constitute a dynamic process of continued adjustment and optimization. When the deviations between the opinions of all individual DMs and the group consensus fall within a reasonable range, we can think that the group has reached a basic consensus (an acceptable consensus). When seen from the angle of mathematical optimization, an acceptable consensus represents the Pareto optimization (Chankong and Haimes, 1983) of the individuals' opinions and the opinion of the moderator. The following diagram shows the step of opinion optimization by the moderator:

Evidently, reaching an acceptable consensus, in terms of mathematical optimization, embodies such a process that the deviations between the opinions of individual DMs and the group opinion are gradually reduced. So, one of the problems this paper needs to address is how to construct such a mathematical optimization model that can be utilized to minimize the deviations between the opinions of individual DMs and the group opinion so that an acceptable consensus can be obtained. 
For example, if the moderator selects the weighted arithmetic averaging operator, then the decision rule will be the weighted arithmetic average principle. Let $F$ satisfy

$$
F\left(o_{1}, o_{2}, \cdots, o_{m}\right)=O W A\left(o_{1}, o_{2}, \cdots, o_{m}\right)=\sum_{t=1}^{m} \omega_{t} o_{\sigma_{t}}
$$


where $\left\{\sigma_{1}, \sigma_{2}, \ldots, \sigma_{m}\right\}$ is a permutation of $\{1,2, \ldots, m\}$. Then Eq. (1) denotes the (aggregated) collective opinion. If $i=\sigma_{i}, i \in M=\{1,2, \ldots, m\}$, i.e.

$$
F\left(o_{1}, o_{2}, \cdots, o_{m}\right)=\sum_{t=1}^{m} \omega_{t} o_{t}
$$

then $F$ is known as a weighted arithmetic average operator. If $\left|o_{\sigma_{(i-1)}}\right| \geq\left|o_{\sigma_{(i)}}\right|, i=2,3, \ldots, m$, then $F$ is known as an ordered weighted (arithmetic) aggregation (OWA) operator.

Additionally, we like to mention that the classical Arrow's work (Arrow, 1963) deals with the aggregation of individual ordinal preferences, while this paper deals with the aggregation of individual cardinal preferences.

\subsection{Basic Hypothesis on Consensus Decision Making}

According to Eqs. (1) - (2), an aggregation operator is actually a function of the weights and opinions of the individuals because the result of aggregation changes with the weights and opinions. Additionally, an appropriate aggregation operator can fully integrate all individuals' opinions and contribute to the objectivity and correctness of the collective opinion. In this sense, selecting the best aggregation operator is also an optimization process.

Conclusively, the optimization model of consensus is based on the following hypotheses: (1) The opinions of individual DMs are assimilated through incessant adjustment and revision of many rounds. (2) The applied aggregation operator is the optimum or could at least fully integrate all individuals' opinions through recurrent selection. And (3) the decision ability of the group is reflected by the individuals' opinions to some extent and in consequence, the weights of the individuals show the objectivity according to hypothesis (1). According to hypothesis (2), the more appropriate the applied aggregation operator is, the higher consensus is (the smaller difference between individuals' 


\section{Optimization Models of Collectively Acceptable Consensus Based on OWA Operator and Their Properties}

In this section, we first introduce an optimization consensus model based on the minimum consensus deviation and investigate the economic meaning of this optimization model. Then we generalize this optimization consensus model to the case of ordered weighted aggregation (OWA) operator, and discuss the economic meaning of the optimization model.

\subsection{The Principle of Constructing the Optimization Consensus Model Based on the Optimal Resource Reallocation}

\subsubsection{The Principle of Constructing the Model}

Let $o_{i}$ be the opinion of $\mathrm{DM} d_{i}, i \in M=\{1,2, \ldots, m\}$. In consensus decision making, when all the opinions are equal to the same ideal opinion $\bar{o}$, the group arrives at a completely consensus. That is, $o_{1}=o_{2}=\ldots=o_{m}=\bar{o}$. Such an ideal opinion actually represents the collective interest, so we refer to it as a consensus opinion. In reality, there must exist a deviation between an individual opinion $o_{i}$ and the consensus opinion $\bar{o}$. This deviation can be expressed by the deviation measure $e_{i}=o_{i}-\bar{o}$. In GDM, when all values of the deviation measure attain the minimum possible value 0 , that is, when the multi-objective optimization problem $\operatorname{Min} e=\left(e_{1}, e_{2}, \ldots, e_{m}\right)^{T}$ has optimal solutions, the group arrives at a completely consensus. If $\left|e_{i}\right|=\left|o_{i}-\bar{o}\right|$, then $\left|e_{i}\right|$ denotes the distance measure between the individual opinion $o_{i}$ and the consensus opinion $\bar{o}$. Similarly, in 
GDM, when all the values of distance measure attain the minimum possible value, that is, when the multi-objective optimization problem (Chankong and Haimes, 1983) Min $|e|=\left(\left|e_{1}\right|,\left|e_{2}\right|, \ldots,\left|e_{m}\right|\right)^{T}$ has optimal solutions, the group also arrives at a complete consensus. Based on the multi-objective optimization theory, it is hard to obtain the optimization solution of a multi-objective optimization problem, and all multi-objective optimization problems need to be transformed into a single-objective model (Chankong and Haimes, 1983) in terms of a decision rule, say, the weighted arithmetic average. This means that we can only obtain the Pareto optimization solutions to $\operatorname{Min} e=\left(e_{1}, e_{2}, \ldots, e_{m}\right)^{T}$ and $\operatorname{Min}|e|=\left(\left|e_{1}\right|,\left|e_{2}\right|, \ldots,\left|e_{m}\right|\right)^{T}$. This also signifies the fact that it is only feasible to obtain the Pareto optimization consensus opinion that represents each individual DM's interest.

For the purpose of arriving at a consensus, the moderator in GDM is entrusted with his ability to persuade each individual DM to modify his/her opinion towards a consensus opinion by paying a price to the DM. When the individual DMs need to change their opinions towards a consensus opinion, it is assumed that they expect to receive appropriate returns or compensations for their adoptions of new positions and opinions. That is, when these individual DMs change their opinions, the moderator pays for their loss according to the deviation degree $\left|e_{i}\right|$. Let $\omega_{0}$ be the total cost that the moderator paid for reaching a consensus, and $\omega_{i}$ the unit cost that the moderator is willing to pay DM $d_{i}$ to achieve a consensus. Then $w_{i}\left|e_{i}\right|$ denotes the total cost paid to DM $d_{i}$. Let $C=\left(\omega_{1}\left|e_{1}\right|, \ldots, \omega_{m}\left|e_{m}\right|\right)^{T}$ denote the total cost that the moderator pays all the individual DMs. Then it is obvious that

$$
\omega_{0}=\sum_{i=1}^{m} \omega_{i}\left|e_{i}\right|
$$

Economically, Eq.(3) possesses the following vital significance. It can be regarded as a reallocation of the limited resource $\omega_{0}$ for consensus reaching: the moderator pays each DM the unit cost $\omega_{i}$ in the light of the distance deviation $\left|e_{i}\right|$ between the DM's opinion $o_{i}$ and the consensus opinion $\bar{o}$, and the total cost spent on DM $d_{i}$ is $\omega_{i}\left|e_{i}\right|$.

If we normalize the unit cost $\omega_{i}$ by $\omega_{i}=\omega_{i} / \sum_{i=1}^{m} \omega_{i}$, the Eq.(3) is equivalent to

$$
\omega_{0}=\sum_{i=1}^{m} \omega_{i}\left|e_{i}\right|
$$

where $\omega_{0}=\omega_{0} / \sum_{i=1}^{m} \omega_{i}$, and $\sum_{i=1}^{m} \omega_{i}=1$.

Similarly, Eq.(4) has the same economic meaning of reaching consensus: It can be regarded as a reallocation of limited resource $\omega_{0}$, where the moderator pays DM $d_{i}$ unit cost $\omega_{i}$ in the light of the distance deviation $\left|e_{i}\right|$ between the DM's opinion $o_{i}$ and the consensus opinion $\bar{o}$, and the total cost on $d_{i}$ is $\omega_{i}\left|e_{i}\right|$. 
For Eq.(4), it is natural to discuss what is the appropriate value of $\omega_{i}$ so that the reallocation of limited resource $\omega_{0}$ is reasonable in the process of reaching a consensus? From the view point of DM $d_{i}, \omega_{i}$ is his unit compensation paid by the moderator according to the deviation value $e_{i}$; from the view point of mathematics, $\omega_{i}$ satisfies $\sum_{i=1}^{m} \omega_{i}=1$, so $\omega_{i}$ can be viewed as the weight of $\left|e_{i}\right|$. Therefore, one of the main purposes of this paper is to determine the objective value of $\omega_{i}$.

Let's reconsider Eq.(4). It satisfies the inequality $\left|\sum_{i=1}^{m} \omega_{i} e_{i}\right| \leq \sum_{i=1}^{m} \omega_{i}\left|e_{i}\right|$, where $\sum_{i=1}^{m} \omega_{i} e_{i}$ is the sum of all the weighted arithmetic average of consensus deviations between the DMs' opinions and the consensus opinion. So, we have

$$
\sum_{i=1}^{m} \omega_{i} e_{i}=\sum_{i=1}^{m} \omega_{i}\left(o_{i}-\bar{o}\right)=\sum_{i=1}^{m} \omega_{i} o_{i}-\bar{o}
$$

If the GDM arrives at a complete consensus, then all the individual DMs' opinions are exactly equal to the consensus opinion (the ideal opinion), i.e., $\bar{o}=o_{1}=o_{2}=\ldots=o_{m}$. For the reason that $\bar{o}=\sum_{i=1}^{m} \omega_{i} \bar{o}=\sum_{i=1}^{m} \omega_{i} o_{i}$, it means that the consensus opinion can be decomposed into the weighted arithmetic average of all the DMs' individual opinions, and it also signifies that the consensus opinion is actually determined by combining individual DMs' opinions. However, in most practical situations, there always exists a deviation between the consensus opinion and the combination of individual DMs' opinions. This means that $\bar{o}$ is not equal to $\sum_{i=1}^{m} \omega_{i} o_{i}$. So, $\sum_{i=1}^{m} \omega_{i} o_{i}-\bar{o}=\sum_{i=1}^{m} \omega_{i} e_{i}$ can be actually viewed as a measure for the degree of deviation from the collective consensus. The smaller the value of this measure is, the greater the degree of consensus.

\subsubsection{Model Construction}

When $\left|\sum_{i=1}^{m} \omega_{i} e_{i}\right|$ attains the minimum value on the universe of discourse $\left\{\omega_{i} \mid \sum_{i=1}^{m} \omega_{i}=1, \omega_{i} \geq 0\right\}$, the GDM reaches the greatest degree of consensus, which can be denoted by the optimization model

$$
\begin{aligned}
& E(\Omega)=\min \left|\sum_{t=1}^{m} \omega_{t} e_{t}\right| \\
& \text { s.t. }\left\{\sum_{t=1}^{m} \omega_{t}=1, \omega_{t} \geq 0, t \in M\right.
\end{aligned}
$$

Obviously, the feasible solution $\omega_{i}, i \in M$, and the optimization solution $\omega_{i}^{*}, i \in M$, to Model (5) satisfy

$$
\begin{gathered}
\min \left|\sum_{i=1}^{m} \omega_{i} e_{i}\right| \leq\left|\sum_{i=1}^{m} \omega_{i} e_{i}\right| \leq \sum_{i=1}^{m} \omega_{i}\left|e_{i}\right| \\
\left|\sum_{i=1}^{m} \omega_{i}^{*} e_{i}\right|=\min \left|\sum_{i=1}^{m} \omega_{i} e_{i}\right| \leq \sum_{i=1}^{m} \omega_{i}^{*}\left|e_{i}\right|
\end{gathered}
$$

where $\omega_{i}, i \in M$, and $\omega_{i}^{*}, i \in M$. Eq. (6) and Eq. (7) have two important meanings: 
- Theoretically, $\omega_{i}$ is the weight of $e_{i}$ and $\omega_{i}^{*}$ the optimal weight of $e_{i}$;

- Practically, $\omega_{i}$ is the unit cost that the moderator paid the DM $d_{i}$; And moreover, if $\left|\sum_{i=1}^{m} \omega_{i} e_{i}\right|$ attains the minimum value under the condition that $\sum_{i=1}^{m} \omega_{i}=1, \omega_{i} \geq 0$, then $\omega_{i}^{*}$ can be regarded as a Pareto optimal reallocations of limited resource $\omega_{0}=\sum_{i=1}^{m} \omega_{i}^{*}\left|e_{i}\right|$ to DM $d_{i}$ for reaching the greatest consensus.

Naturally, we need to discuss the distribution of weights by solving the optimization Model (5). Similarly, Model (5) also has two important meanings:

- It is an optimal consensus model under the condition $\sum_{i=1}^{m} \omega_{i}=1, \omega_{i} \geq 0$; and

- It is an optimal resource reallocation model of the limited resource $\omega_{0}=\sum_{i=1}^{m} \omega_{i}^{*}\left|e_{i}\right|$ for reaching the greatest consensus.

In the next section, a more general optimization consensus model is developed based on Model (5).

\subsection{A Generalized Optimization Consensus Model Based on the Optimal Resource Reallocation}

Consider the set $\left\{e_{1}, e_{2}, \ldots, e_{m}\right\}$ of individual deviation measures, and the corresponding $\left\{\omega_{1}, \omega_{2}\right.$, $\left.\ldots, \omega_{m}\right\}$ set of weights, satisfying $\sum_{t=1}^{m} \omega_{t}=1,0 \leq \omega_{t} \leq 1$. Suppose that the aggregation operator of the decision rule is the ordered weighted (arithmetic) aggregation (OWA). The degree of collective consensus deviation $\sum_{t=1}^{m} \omega_{t} e_{\sigma_{t}}$ is obtained by integrating all DMs' opinions using the OWA operator, where $\left\{\sigma_{1}, \sigma_{2}, \ldots, \sigma_{m}\right\}$ is a permutation of $\{1,2, \ldots, m\}$, and $e_{\sigma_{t-1}} \geq e_{\sigma_{t}}, t=2,3, \ldots, m$, and the $O W A$ operator (Yager, 1988) of the dimension $m$ is a function $O W A: R^{n} \mapsto R$ with an associated weight vector $\Omega=\left(\omega_{1}, \omega_{2}, \ldots, \omega_{m}\right)^{T}$ such that $\sum_{t=1}^{m} \omega_{t}=1$. Let

$$
E=o-\bar{o}=\sum_{t=1}^{m} \omega_{t} e_{\sigma_{t}}
$$

Then $E$ is actually a combination of individual consensus (deviation) levels, and also a function of the objective weight vector $\Omega=\left(\omega_{1} \ldots \omega_{m}\right)^{T}$. So we denote $E$ as a function $E(\Omega)$, where $\left\{\sigma_{1}, \sigma_{2}, \ldots, \sigma_{m}\right\}$ is a permutation of $\{1,2, \ldots, m\}$, and $o_{\sigma_{t-1}} \geq o_{\sigma_{t}}, t=2,3, \ldots, m$. Here, we define $|E|$ to be the collective consensus (deviation) level. Obviously, the closer to 0 the function $E(\Omega)$ is, the larger the collective consensus level is. 
Suppose that there is a threshold value $\varepsilon, \varepsilon \geq 0$, such that the equation

$$
\left|e_{\sigma_{t}}\right|=\left|\bar{o}-o_{\sigma_{t}}\right| \leq \varepsilon \text { or } o_{\sigma_{t}}-\varepsilon \leq \bar{o} \leq o_{\sigma_{t}}+\varepsilon, t \in M
$$

holds true. Then we say that the DMs in the GDM reach an individually acceptable consensus. Next, we show that for any established threshold $\varepsilon, \varepsilon \geq 0$, the distance $|E|$ is also within the interval $[0, \varepsilon]$. It is readily to prove that

$$
|E| \leq \varepsilon
$$

If Eq. (10) holds true, we say that the DMs in the GDM reach a collectively acceptable consensus. The following theorem is readily seen.

Theorem 1 For any given threshold value $\varepsilon, \varepsilon \geq 0$, if the DMs in the GDM reach the individually acceptable consensus, then the DMs in the GDM also reach the collectively acceptable consensus.

In this paper, we call $\varepsilon(\varepsilon \geq 0$ satisfies Eq.(9)) the threshold value of acceptable consensus. Obviously, the smaller the threshold value of acceptable consensus is, the higher individual consensus level and collective consensus level are.

\subsubsection{The Optimization Consensus Model Based on OWA Operator}

Grounded on the previous analysis, the smaller the function $E(\Omega)$ is, the larger the collective consensus level is. The function $E(\Omega)$ of the collective consensus (deviation) level (the objective function) should be the minimum under the condition of linear constraint $\omega_{1}+\ldots+\omega_{m}=1, \omega_{i} \geq 0$. Thus we have the following optimization consensus model

$$
\begin{aligned}
& E(\Omega)=\min O W A\left(e_{1}, e_{2}, \ldots, e_{m}\right)=\min \left|\sum_{t=1}^{m} \omega_{t} e_{\sigma_{t}}\right| \\
& \text { s.t. }\left\{\sum_{t=1}^{m} \omega_{t}=1, \omega_{t} \geq 0, t \in M\right.
\end{aligned}
$$

where $\left\{\sigma_{1}, \sigma_{2}, \ldots, \sigma_{m}\right\}$ is a permutation of $\{1,2, \ldots, m\}$, and $e_{\sigma_{t-1}} \geq e_{\sigma_{t}}, t=2,3, \ldots, m$.

We call Model (11) the optimization consensus model based on OWA operator (COWA Model). The optimization solution to Model (11) is beneficial to the investigation of the economic meaning and the systemic meaning of the weight vector. Moreover, we will also discuss when new opinions are added into the GDM, whether or not the level of consensus will be changed and what is the economic meaning of this situation?

\subsubsection{The Generalized Optimization Consensus Model Based on the Optimal Resource Reallocation}

If we let $\omega_{i}$ be the unit cost that the moderator paid DM $d_{\sigma_{i}}$, where $\left\{\sigma_{1}, \sigma_{2}, \ldots, \sigma_{m}\right\}$ is a permutation of $\{1,2, \ldots, m\}$, then $\omega_{i}$ in Model (11) has two important meanings: 
- Theoretically, $\omega_{i}$ is the weight of $e_{\sigma_{i}}$; and

- Practically, $\omega_{i}$ is the unit cost that the moderator paid DM $d_{\sigma_{i}}$; Moreover, if $\left|\sum_{i=1}^{m} \omega_{i}^{*} e_{\sigma_{i}}\right|$ attains the minimum value under the condition $\sum_{i=1}^{m} \omega_{i}^{*}=1, \omega_{i}^{*} \geq 0$, then $\omega_{i}^{*}$ can be regarded as an Pareto optimal reallocations of limited resource $\omega_{0}=\sum_{i=1}^{m} \omega_{i}^{*}\left|e_{\sigma_{i}}\right|$ for reaching greatest consensus.

Naturally, the weight $\omega_{i}$ in Model (11) is the main points of the discussion. And Model (11) also has two important meanings:

- It is an optimal consensus model under the condition $\sum_{i=1}^{m} \omega_{i}^{*}=1, \omega_{i}^{*} \geq 0$; and

- It is an optimal resource reallocation model of limited resource $\omega_{0}=\sum_{i=1}^{m} \omega_{i}^{*}\left|e_{\sigma_{i}}\right|$ for reaching greatest consensus.

Let's reconsider the economic meaning of

$$
\omega_{0}=\sum_{i=1}^{m} \omega_{i}\left|e_{\sigma_{i}}\right|
$$

It can be regarded as a reallocation of the limited resource $\omega_{0}$ : the moderator pay the DM $d_{\sigma_{i}}$ the unit cost $\omega_{i}$ in light of the deviation between the DM's opinion $o_{\sigma_{i}}$ and the consensus opinion $\bar{o}$, and the total cost paid to the individual $d_{\sigma_{i}}$ is $\omega_{i}\left|e_{\sigma_{i}}\right|$.

For a given threshold value of acceptable consensus $\varepsilon, \varepsilon \geq 0,\left|e_{\sigma_{i}}\right| \leq \varepsilon$, we have

$$
\begin{gathered}
\omega_{i}\left|e_{\sigma_{i}}\right| \leq \omega_{i} \varepsilon \\
E \leq \omega_{0} \leq \varepsilon
\end{gathered}
$$

The economic meaning of Eq. (13) and Eq. (14) is as following: The limited resource $\omega_{0}$ is no less than the minimum value of reaching consensus $E$, and no more than the given threshold value of acceptable consensus $\varepsilon$. This means that $E$ is the lower bound of limited resource $\omega_{0}$, and $\varepsilon$ the upper bound of $\omega_{0}$. Additionally, the total cost paid to DM $d_{\sigma_{i}}$ is no more than $\omega_{i} \varepsilon$.

\subsection{Particular Optimization Solutions to the COWA Model}

In this section, we further investigate the economic meanings and systemic significance of the weights in Model (11) by discussing its particular optimal solutions.

Let $\varepsilon \geq 0$ be a given threshold value of acceptable consensus satisfying Eq.(9), and $\Omega^{*}=$ $\left(\omega_{1}^{*} \omega_{2}^{*} \ldots \omega_{m}^{*}\right)^{T}$ satisfying $\omega_{1}^{*}+\omega_{2}^{*}+\ldots+\omega_{m}^{*}=1$, the optimal solution vector to the COWA model. If $E\left(\Omega^{*}\right)=\min E(\Omega)$, then the COWA model is referred to as the optimal consensus model, 
$\Omega^{*}$ the optimal solution vector to the COWA model, and $E\left(\Omega^{*}\right)$ collective (Pareto) optimal consensus (deviation) level.

Theorem 2 Let $\sum_{t=1}^{m} e_{t}=0$. Then $\Omega=(1 / m, 1 / m, \ldots, 1 / m)^{T}$ is an optimal solution to the COWA Model, such that $E(\Omega)=0$.

The economic meaning of Theorem 2 is that when the sum of all individual deviations is complementary (equivalent to 0), the degree of consensus is the highest and the moderator pays each DM the same unit price. In this situation, the decision-making group may have a unanimous consent on the issue of concern. In other words, if all individual deviations balance each other out, we regard the importance of all individual DMs as being the same. The following entropy theorem shows that this GDM system is stable.

Information entropy, as introduced by Shannon in 1948 (Shannon, 1948), is used to measure the degree of uncertainty or information quantity of random events. In a consensus decision making system, the weight information of all the DMs can be regarded as a random variable $\Omega=\left\{\omega_{1}, \omega_{2}, \ldots, \omega_{m}\right\}$, where $\omega_{i}$ is the weight of the DM $d_{i}, i \in M$. Each weight $\omega_{i}, i \in M$, can be viewed as independent while satisfying $\sum_{i=1}^{m} \omega_{i}=1$. Then $\left\{\omega_{1}, \omega_{2}, \ldots, \omega_{m}\right\}$ is in fact a probability distribution. In light of the maximum entropy principle, when the system's entropy reaches the maximum, that is, when

$$
s=-\sum_{i=1}^{m} \omega_{i} \ln \omega_{i}
$$

reaches the maximum, where $\sum_{i=1}^{m} \omega_{i}=1$, the system is the most stable and the random variable is optimal.

In Eq.(15), when $\omega_{1}=\omega_{2}=\ldots=\omega_{m}$, the entropy model reaches the maximum, the unit cost paid to each individual DM is the same, and the stability of the consensus decision making is the highest. When there is a weight $\omega_{i}=1$, and the rest weights $\omega_{j}=0, j \neq i, j \in M$, the entropy model reaches the minimum value, and the stability of consensus decision making is the lowest.

The entropy model (15) of consensus decision making and Theorem 2 can be interpreted as that there is no difference in the importance of each DM (that is, the unit cost paid to each individual DM is the same) so that the collective opinion possesses higher objectivity, and that the decision system is the most stable (All deviations of the individuals' opinions can balance each other out. That is, the sum of the deviations is 0.). So, consequently, the GDM reaches the highest consensus. Additionally, the variance of a weight variable is also an important index that can be employed to measure the stability of the GDM system. The concept of variance of a random variable measures the spread, or variability of a distribution. In Theorem 2 , when the sum of all the individual deviation is 0 , the variance of all weights is 0 . This shows that the spread, or variability, of the weights of the DMs is 
minimum. All in all, when the individuals' deviations balance each other out, we have that

- the moderator pays each DM the same unit cost in terms of the economic meaning; and

- the decision making system is most stable.

Next, we prove that when the individuals' deviations $e_{t}, t \in M$, are all positive or all negative, the COWA Model has an optimal solution.

Theorem 3 Let $e_{t}, t \in M$, be positive (respectively, negative), $\Omega_{m}=\left(\begin{array}{lllll}0 & 0 & \ldots & 0 & 1\end{array}\right)^{T}$ an optimal solution to the COWA Model, where $\omega_{m}=1, \omega_{i}=0, i \neq m, i \in M$, and $\left|e_{m}\right|=e_{\min }=\min \left\{\left|e_{t}\right|, t \in\right.$ $M\}$ denotes the minimum level of individual consensus deviations.

Theorem 3 shows that when all DMs' opinions are greater than (respectively, smaller than) the consensus opinion $\left(e_{t}>0, t \in M\right)$; or when all DMs' opinions are smaller than the consensus opinion $\left(e_{t}<0, t \in M\right)$, the DM whose individual consensus level is the highest is optimal. Theorem 3 also explains that all the other individuals' opinions are redundant except for the opinion of DM $d_{\sigma_{m}}$. In other words, $d_{\sigma_{m}}$ obtains all the compensation, while the rest DMs receive nothing. That means that the information entropy model (15) reaches the minimum value. In light of the information entropy principle, the minimum information entropy has the weakest stability. Additionally, in Theorem 3 , when $e_{t}, t \in M$, are all positive (respectively, negative), the variance of all weights attains the maximum value. This shows that the spread, or variability, of the weights of the DMs is also maximum.

In conclusion, when all the other individuals' opinions are redundant except for one opinion, we have

- only one DM presents useful opinion, and the moderator pay all the cost to this individual;

- this decision making system has the weakest stability.

\subsection{Special Properties of the Optimization Model of Acceptable Consensus When New DMs Join}

In this section, we will show that when we add additional DMs to the GDM, the consensus level based on the COWA model will not decrease, and the moderator's total cost on all DMs for changing their individual DMs' opinions towards the consensus opinion is also no more than a fixed value.

Theorem 4 If $q, q \geq 1$, additional DMs are added to the optimal value of the COWA Model, then the collective consensus level does not decrease. That is,

$$
E^{*}\left(\omega_{1}, \omega_{2}, \ldots, \omega_{m+q}\right) \leq E^{*}\left(\omega_{1}, \omega_{2}, \ldots, \omega_{m}\right)
$$


where $E^{*}\left(\omega_{1}, \omega_{2}, \ldots, \omega_{m+q}\right)$ and $E^{*}\left(\omega_{1}, \omega_{2}, \ldots, \omega_{m}\right)$ are the optimal objective functions of the COWA Model.

This conclusion can explain that in a fair play, such as the athletic competitions as gymnastics or diving, program reviews, the more DMs take relatively rational decisions, then the larger number of DMs needs to be involved, and the more impartial are the decision results. For one example, in a diving or a gymnastics competition, the referees score the athletes from different flanks and angles. If we regard the importance of all the referees as objective and relatively rational, then the larger number of the referees, the fairer of the evaluation of the athletes.

Corollary 1a (The consensus meaning) For any given threshold value of acceptable consensus $\varepsilon, \varepsilon \geq 0$, if the DMs in the GDM reach a collective acceptable consensus, then when more than one DMs are added into the GDM, the consensus level based on the COWA model is still acceptable.

Corollary 1b (The economic meaning) For any given threshold value of acceptable consensus $\varepsilon, \varepsilon \geq 0$, if the DMs in the GDM reach a collective acceptable consensus, then when more than one DMs are added into the GDM, the moderator's cost is no more than the threshold value of acceptable consensus.

Theorems 4 shows that once the rational (ideal) opinion is determined, the consensus level based on the COWA model is still acceptable whenever one additional DM is added into the GDM each time for many times or more than one DM are added into the GDM at once.

Connotations of Theorems 4 and Corollaries 1 include that the collective consensus level is a nonincreasing function of the number of DMs under the objective of minimizing the consensus deviation. This conclusion can explain that in such situations as program reviews, parliamentary elections, and international negotiations, when new opinions join the GDM, as long as the opinions are acceptable, the consensus level will not decrease, and the moderator's cost on all DMs is no more than a fixed upper limitation (the threshold value of acceptable consensus).

Theorem 5 If $\sum_{t=1}^{m} e_{t}=0$, for all $m \in Z^{+}=\{1,2, \cdots\}$, then the entropy of the GDM system based on the COWA model increases with the number of DMs.

Theorem 5 indicates that when all the individuals' deviations in the GDM balance each other out, the larger the number of the DMs is, the more stable the GDM system is. The economic meaning of Theorem 5 is that even with additional DMs entering into the GDM, as long as the condition $\sum_{t=1}^{m} e_{t}=0$, holds true for all $m \in Z^{+}=\{1,2, \cdots\}$, the GDM system continues to be stable, and the moderator's cost on all DMs is no more than a fixed upper limitation (the moderator does not need to pay extra). 


\section{Numerical Examples and An Instance: Demolition and Reloca- tion During Urbanization Process}

In this section, three numerical examples are firstly used to illustrate that entropy and variance are two important metrics to measure the stability of the GDM system; then, an instance about the demolition and relocation during urbanization process is adopted to further explain the economic significance of model (11), which also verifies the rationality of Corollary 1a and 1b.

\subsection{Numerical Examples}

Example 1 For a GDM problem, assume that $D=\left\{d_{1}, d_{2}, \ldots, d_{8}\right\}$ is a set of DMs, and $\Omega=$

$\left(\omega_{1}, \omega_{2}, \ldots, \omega_{8}\right)^{T}$ the corresponding weight vector, satisfying $\sum_{t=1}^{8} \omega_{t}=1,0 \leq \omega_{t} \leq 1$. Let the set of the individual real opinions be

$$
o=\left\{o_{1}, o_{2}, \cdots, o_{8}\right\}=\{9.2,9.2,10.5,10.5,10.8,10.8,9.8,9.8\}
$$

the collective rational opinion be 10 and the consensus threshold value is $\varepsilon=0.01$. Then the consensus deviations are $\{-0.8,-0.8,0.5,0.5,0.8,0.8,-0.2,-0.2\}$.

According to Model (11), the optimization consensus model is constructed as follows.

$$
\left\{\begin{aligned}
O(\Omega) & =\min |O W A(-0.8,-0.8,0.5,0.5,0.8,0.8,-0.2,-0.2)| \\
& =\min \left|0.8 \omega_{1}+0.8 \omega_{2}+0.5 \omega_{3}+0.5 \omega_{4}-0.2 \omega_{5}-0.2 \omega_{6}-0.8 \omega_{7}-0.8 \omega_{8}\right| \\
\text { s.t. } & \omega_{1}+\omega_{2}+\omega_{3}+\omega_{4}+\omega_{5}+\omega_{6}+\omega_{7}+\omega_{8}=1, \omega_{t} \geq 0, t=1,2, \ldots, 8
\end{aligned}\right.
$$

The optimal solution to Model (16) is

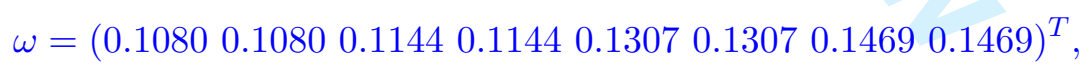

which denotes that the weights vector of the 8 decision makers is

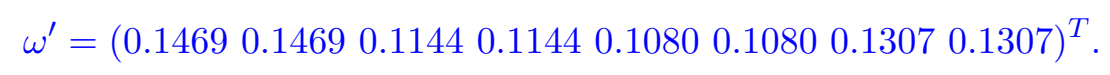

The collective OWA opinion is

$0.8 * 0.1080+0.8 * 0.1080+0.5 * 0.1144+0.5 * 0.1144-0.2 * 0.1307-0.2 * 0.1307-0.8 * 0.1469-0.8 * 0.1469=10$,

which is equivalent to the consensus rational opinion 10. In this example, all the weights is close to the average weight 0.125 , and the entropy of the GDM is $-0.1469 * \ln (0.1469)-0.1469 * \ln (0.1469)-$ $0.1144 * \ln (0.1144)-0.1144 * \ln (0.1144)-0.1080 * \ln (0.1080)-0.1080 * \ln (0.1080)-0.1307 * \ln (0.1307)-$ $0.1307 * \ln (0.1307)=2.0722$. 
Example 2 Suppose that there are two DMs in a GDM and the consensus deviation vector is $C_{2}=\left(\begin{array}{ll}-0.8 & 0.5\end{array}\right)^{T}$. We add one DM to this GDM system each time for eight times. Then we get a series of GDM systems with $i$ DMs, $i=2 \ldots, 10$, and assume that the corresponding consensus deviation vectors, as shown in Table 1.

Table 1: The deviation vectors of all GDM systems

\begin{tabular}{c|c}
\hline$c_{2}$ & $(-0.80 .5)$ \\
$c_{3}$ & $(-0.80 .50 .5)$ \\
$c_{4}$ & $(-0.80 .50 .5-0.2)$ \\
$c_{5}$ & $(-0.80 .50 .5-0.2-0.2)$ \\
$c_{6}$ & $(-0.80 .50 .5-0.2-0.2-0.2)$ \\
$c_{7}$ & $(-0.80 .50 .5-0.2-0.2-0.20 .3)$ \\
$c_{8}$ & $(-0.80 .50 .5-0.2-0.2-0.20 .30 .3)$ \\
$c_{9}$ & $(-0.80 .50 .5-0.2-0.2-0.20 .30 .3-0.4)$ \\
$c_{10}$ & $(-0.80 .50 .5-0.2-0.2-0.20 .30 .3-0.40 .9)$ \\
\hline
\end{tabular}

Similar to Example 1, we get the weight vector, the variance, and the entropy of each GDM system as shown in Table 2 and Table 3.

Table 2: The objective weight vectors of all GDM systems

\begin{tabular}{|c|c|}
\hline$X_{2}$ & $(0.38460 .6154)^{T}$ \\
\hline$X_{3}$ & $\left(\begin{array}{lll}0.3846 & 0.3077 & 0.3077\end{array}\right)^{T}$ \\
\hline$X_{4}$ & $\left(\begin{array}{lllll}0.2500 & 0.2500 & 0.2500 & 0.2500\end{array}\right)^{T}$ \\
\hline$X_{5}$ & $\left(\begin{array}{lllll}0.1735 & 0.2172 & 0.2172 & 0.1961 & 0.1961\end{array}\right)^{T}$ \\
\hline$X_{6}$ & 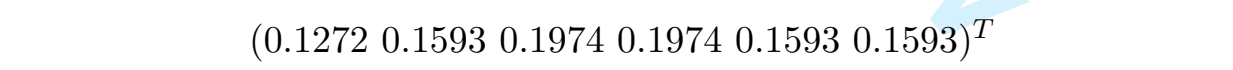 \\
\hline$X_{7}$ & 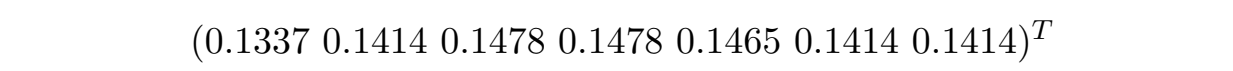 \\
\hline$X_{8}$ & 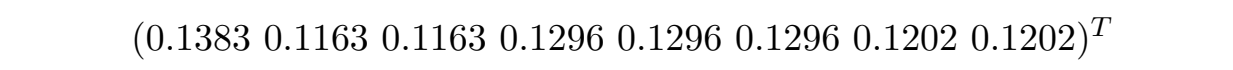 \\
\hline$X_{9}$ & 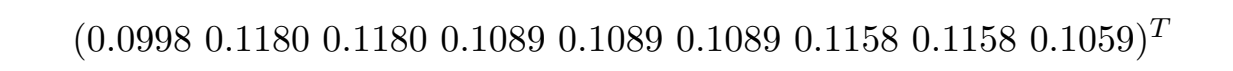 \\
\hline$X_{10}$ & 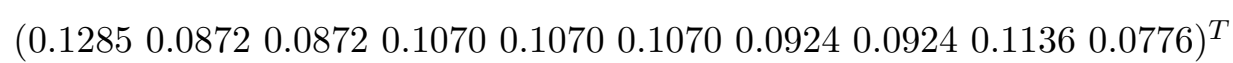 \\
\hline
\end{tabular}

In Example 2, we see that the deviation values in each GDM system are relatively well distributed, and that the information entropy (and the variance) of weight vectors in each GDM system have the tendency to increase (decrease) with the number of the DMs involved in the decision process. This shows that the stability of a GDM increases with the number of the DMs.

Example 3 Suppose that there are two DMs in a GDM and the consensus deviation vector is 
Table 3: The optimal consensus deviations, variances and the entropies of all GDM systems

\begin{tabular}{c|ccccccccc}
\hline$C$ & $c_{2}$ & $c_{3}$ & $c_{4}$ & $c_{5}$ & $c_{6}$ & $c_{7}$ & $c_{8}$ & $c_{9}$ & $c_{10}$ \\
\hline$X$ & $X_{2}$ & $X_{3}$ & $X_{4}$ & $X_{5}$ & $X_{6}$ & $X_{7}$ & $X_{8}$ & $X_{9}$ & $X_{10}$ \\
\hline$E$ & $E_{2}^{\prime}=0$ & $E_{3}^{\prime}=0$ & $E_{4}^{\prime}=0$ & $E_{5}^{\prime}=0$ & $E_{6}^{\prime}=0$ & $E_{7}^{\prime}=0$ & $E_{8}^{\prime}=0$ & $E_{9}^{\prime}=0$ & $E_{10}^{\prime}=0$ \\
\hline $\operatorname{Var}(X)$ & 0.0966 & 0.0291 & 0.0125 & 0.0069 & 0.0046 & 0.0026 & 0.0018 & 0.0013 & 0.0011 \\
\hline$-X^{T} * \log (X)$ & 0.6663 & 1.0928 & 1.3863 & 1.6061 & 1.7808 & 1.9454 & 2.0777 & 2.1958 & 2.2922 \\
\hline
\end{tabular}

$C_{2}=\left(\begin{array}{ll}-0.2 & 0.9\end{array}\right)^{T}$. Let us add one DM to this GDM system each time for eight times. Then we get a series of GDM systems with $i$ DMs, $i=2 \ldots, 10$. Assume that the corresponding consensus deviation vectors are shown in Table 4.

Table 4: The deviation vectors of all GDM systems

\begin{tabular}{|c|c|}
\hline$c_{2}^{\prime}$ & $\left(\begin{array}{ll}-0.2 & 0.9\end{array}\right)$ \\
\hline$c_{3}^{\prime}$ & $\left(\begin{array}{lll}-0.2 & 0.9 & 0.9\end{array}\right)$ \\
\hline$c_{4}^{\prime}$ & $\left(\begin{array}{llll}-0.2 & 0.9 & 0.9 & 0.6\end{array}\right)$ \\
\hline$c_{5}^{\prime}$ & $\left(\begin{array}{lllll}-0.2 & 0.9 & 0.9 & 0.6 & 0.7\end{array}\right)$ \\
\hline$c_{6}^{\prime}$ & $\left(\begin{array}{llllll}-0.2 & 0.9 & 0.9 & 0.6 & 0.7 & 0.9\end{array}\right)$ \\
\hline$c_{7}^{\prime}$ & $\left(\begin{array}{lllllll}-0.2 & 0.9 & 0.9 & 0.6 & 0.7 & 0.9 & 0.9\end{array}\right)$ \\
\hline$c_{8}^{\prime}$ & $\left(\begin{array}{lllllllll}-0.2 & 0.9 & 0.9 & 0.6 & 0.7 & 0.9 & 0.9 & 0.9\end{array}\right)$ \\
\hline$c_{9}^{\prime}$ & $\left(\begin{array}{lllllllllll}-0.2 & 0.9 & 0.9 & 0.6 & 0.7 & 0.9 & 0.9 & 0.9 & 0.9\end{array}\right)$ \\
\hline$c_{10}^{\prime}$ & $\left(\begin{array}{lllllllllll}-0.2 & 0.9 & 0.9 & 0.6 & 0.7 & 0.9 & 0.9 & 0.9 & 0.9 & 0.9\end{array}\right)$ \\
\hline
\end{tabular}

Similar to Example 1, we obtain the weight vector, the variance, and the entropy of each of these GDM systems as shown in Table 5 and Table 6.

In this example, we construct a series of extreme GDM systems whose deviation values are not well distributed. That is, there is only a minus deviation value in each GDM system. We show that the stability of a GDM also increase with the number of DMs (as shown in Table 5 and Table 6.).

\subsection{An Instance: Demolition and Relocation During Urbanization Process}

Using the data from Example 1, we take the demolition and relocation during urbanization process for example to further show the economic significance of model (11). That is, using the practical example to illustrate the application background of the optimization model based on acceptable consensus. Meantime, some numerical examples are applied to discuss the effect of adding new DMs on 
Table 5: The objective weight vectors of all the GDM systems

\begin{tabular}{l|c}
\hline$X_{2}^{\prime}$ & $(0.81820 .1818)^{T}$ \\
$X_{3}^{\prime}$ & $(0.81820 .09090 .0909)^{T}$ \\
$X_{4}^{\prime}$ & $(0.79670 .06220 .06220 .0789)^{T}$ \\
$X_{5}^{\prime}$ & $(0.79280 .04800 .04800 .05700 .0542)^{T}$ \\
$X_{6}^{\prime}$ & $(0.79860 .03850 .03850 .04350 .04230 .0385)^{T}$ \\
$X_{7}^{\prime}$ & $(0.80250 .03220 .03220 .03450 .03430 .03220 .0322)^{T}$ \\
$X_{8}^{\prime}$ & $(0.80520 .02750 .02750 .02860 .02870 .02750 .02750 .0275)^{T}$ \\
$X_{9}^{\prime}$ & $(0.80710 .02400 .02400 .02420 .02480 .02400 .02400 .02400 .0240)^{T}$ \\
$X_{10}^{\prime}$ & $(0.80860 .02130 .02130 .02080 .02160 .02130 .02130 .02130 .02130 .0213)^{T}$ \\
\hline
\end{tabular}

Table 6: The optimal consensus deviations, variances and the entropies of the GDM systems

\begin{tabular}{c|ccccccccc}
\hline$C^{\prime}$ & $c_{2}^{\prime}$ & $c_{3}^{\prime}$ & $c_{4}^{\prime}$ & $c_{5}^{\prime}$ & $c_{6}^{\prime}$ & $c_{7}^{\prime}$ & $c_{8}^{\prime}$ & $c_{9}^{\prime}$ & $c_{10}^{\prime}$ \\
\hline$X^{\prime}$ & $X_{2}^{\prime}$ & $X_{3}^{\prime}$ & $X_{4}^{\prime}$ & $X_{5}^{\prime}$ & $X_{6}^{\prime}$ & $X_{7}^{\prime}$ & $X_{8}^{\prime}$ & $X_{9}^{\prime}$ & $X_{10}^{\prime}$ \\
\hline$E^{\prime}$ & $E_{2}^{\prime}=0$ & $E_{3}^{\prime}=0$ & $E_{4}^{\prime}=0$ & $E_{5}^{\prime}=0$ & $E_{6}^{\prime}=0$ & $E_{7}^{\prime}=0$ & $E_{8}^{\prime}=0$ & $E_{9}^{\prime}=0$ & $E_{10}^{\prime}=0$ \\
\hline $\operatorname{Var}\left(X^{\prime}\right)$ & 0.1846 & 0.1453 & 0.1122 & 0.0945 & 0.0838 & 0.0751 & 0.0678 & 0.0618 & 0.0567 \\
\hline$-X^{\prime} * \log \left(X^{\prime}\right)$ & 0.4741 & 0.6002 & 0.7270 & 0.7969 & 0.8261 & 0.8506 & 0.8722 & 0.8916 & 0.9089 \\
\hline
\end{tabular}

the stability of the acceptable consensus system.

In the demolition and relocation project of an urban building, the government (the moderator) needs to persuade the householders (individuals) to move to a new place. In order to acquire more inside information into the matter, the moderator carries out surveys of relocation aspirations. The term (opinion) set of relocation aspirations is designed as follows: Opinion 10 indicates that the individual DM agrees to relocate and the moderator does not need to put in additional effort, thus we define this opinion as a consensus opinion. Obviously, it is also defined as the moderator's opinion. If an individual DM's opinion is lower than 10, it means that, though the individual DM does not agree to relocate, the moderator will try to sway him/her by making the necessary efforts. The lower the opinion is, the stronger desire of obtaining compensation, and the greater effort needs to be made by the moderator. Here, we define any opinion that is under 10 as negative opinion of relocation. On such occasions, the resources consumed by the moderator to sway the individuals are determined by the absolute value of deviation between the negative opinion and the consensus opinion. If an individual DM's opinion is greater than 10, this means that the individual DM agrees to relocate but expect more compensation. In this case, the moderator still needs to pay the individual for his/her loss and for his/her willingness to provide additional support. The greater the opinion is, the stronger 
desire of obtaining compensation, and the greater effort the moderator needs to make. Similarly, we define any opinion that is above 10 as positive opinion of relocation. With these conventions in place, the resources consumed by the moderator to sway the individual DMs are also determined by the absolute value of deviation between the positive opinion and the consensus opinion. The following diagram shows the relation between the desire of obtaining compensation and the opinion of relocation aspirations by the individual DMs.

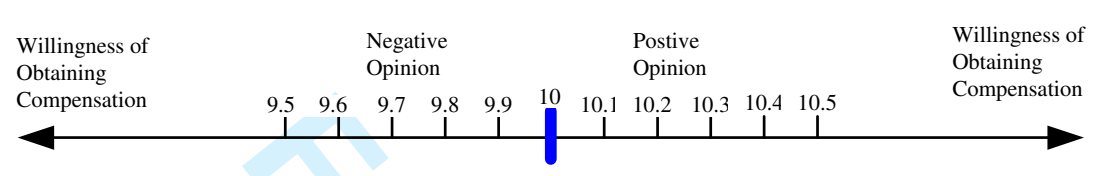

Figure 4: The Relation between the Desire of Obtaining Compensation and the Opinion of Relocation Aspiration

Assume that $D=\left\{d_{1}, d_{2}, \ldots, d_{8}\right\}$ is a set of householders, and

$$
O=\left\{o_{1}, o_{2}, \cdots, o_{8}\right\}=\{9.2,9.2,10.5,10.5,10.8,10.8,9.8,9.8\}
$$

a set of opinions. Suppose that the value of the moderator's (consensus) opinion is 10, then the consensus deviations set is $\{-0.8,-0.8,0.5,0.5,0.8,0.8,-0.2,-0.2\}$. And suppose that the threshold value for reaching a collectively acceptable consensus is $\varepsilon=0.9$, where the DMs in the GDM reach the acceptable consensus. Suppose that $\Omega=\left(\omega_{1} \omega_{2} \ldots \omega_{8}\right)^{T}$ is the corresponding weight vector of the 8 DMs, satisfying $\sum_{t=1}^{8} \omega_{t}=1,0 \leq \omega_{t} \leq 1$. Based on the analysis of Section $3.1, \Omega=\left(\omega_{1} \omega_{2} \ldots \omega_{8}\right)^{T}$ can also be regarded as the unit cost vector that the moderator paid to the individuals. The optimal solution to Model (16) is

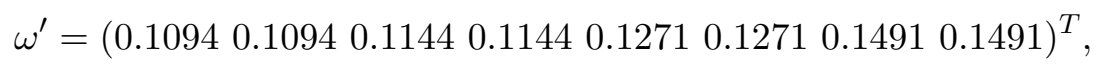

which is also denoted as the unit cost vector that the moderator paid to the 8 DMs. The resources consumed by the moderator to sway the 8 individuals are $0.1193,0.1193,0.0572,0.0572,0.0875,0.0875$, 0.0254, and 0.0254, respectively, and the total 0.5788 for reaching an acceptable consensus is no more than 0.9 . The following diagram shows the respective expenses of the moderator for his effort to sway the 8 individuals.

If there are two more DMs $d_{9}, d_{10}$ entering into this GDM, and the corresponding opinions are respectively 9.9 and 9.8 , then the consensus deviations set is $\{-0.8,-0.8,0.5,0.5,0.8,0.8,-0.2,-0.2,-0.1$, 


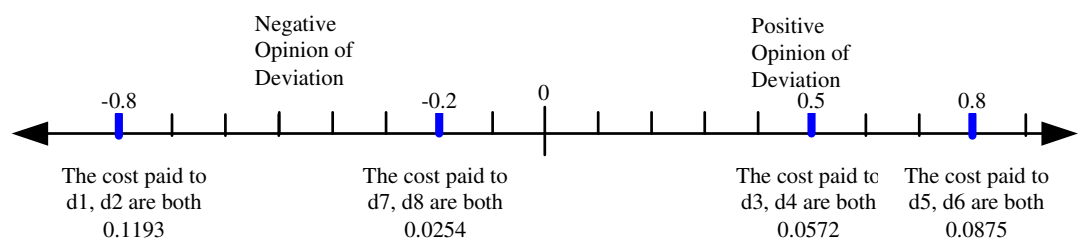

Figure 5: The Cost Paid by the Moderator to the 8 Individuals

$-0.2\}$. By constructing the similar optimization model, the unit cost vector that the moderator paid to the $10 \mathrm{DMs}$ is

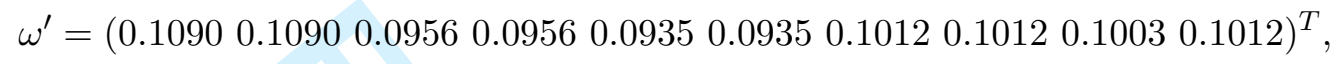

We can also prove that the total cost for reaching an acceptable consensus is no more than 0.9. That is, the moderator does not need to spend anything extra.

\section{Conclusion}

The consensus decision represents the goal of each GDM. This paper first builds the hypothesis that a consensus opinion exists, then regards the weights of individual DMs as objective values. By minimizing the differences between the individuals' opinions and the collective consensus opinion, this paper constructs a consensus optimization model and shows that the objective weights of the individuals are actually the optimal solution to this model. From the point of view of economics, this paper views the optimization model as an optimal resource reallocation model of the limited resource for reaching the greatest consensus, and views the objective weights as the optimal unit cost that the moderator has to pay to the individuals. In particular,

- When there is no difference among all the DMs' weights, and the differences between the individuals' opinions and the consensus opinion can balance each other out, the level of the collective consensus reaches the highest in the GDM system. From the perspective of systems science, this GDM system is the most stable as proved by using the information entropy theory. From the angle of resource reallocation, the moderator pays each individual DM the same unit cost.

- When all individual DMs' real opinions are greater than (respectively, smaller than) the consensus opinion, only the DM whose level of individual consensus deviation is the minimum presents the valuable opinion; the GDM system is the least stable as proved by using the information entropy theory; and the moderator has to pay all the cost to this individual in terms of economics.

- For a given threshold value of acceptable consensus, if the DMs in the GDM has reached a collectively acceptable consensus, then when additional DMs are added into the GDM, the 
consensus level based on the COWA model is still acceptable, and the individuals' compensations are no more than a fixed upper limit.

- When all the individuals' deviations in the GDM balance each other out, even if there are additional DMs entering into the GDM, the GDM system is still acceptable, and the moderator's cost is still no more than a fixed upper limit.

To sum up, in this paper, an optimization model based on acceptable consensus is constructed under the premise of all DMs' weights being objective, and the economic significance of the proposed model, including the theoretical and practical significance, is emphatically analyzed; besides, some properties of the optimization model are discussed by analyzing the particular solutions: the stability of the consensus system is explored through combining DMs' objective weights with information entropy theory and variance distribution; in addition, the effect of adding new DMs on the stability of the acceptable consensus system is explored by studying the convergence of consensus level, which can be seen as the further discussion on the theoretical and economic significance of the optimization model based on acceptable consensus. In this paper, we assume all DMs' weights are completely objective. Actually, in real consensus decision-making, we should take into account that DMs' weights known or partly known. Therefore, modeling the consensus scenarios where DMs' weights known or partly known and exploring their economic significance will be our future research.

\section{References}

Arrow, K.J. (1963), Social Choice and Individual Values, Wiley, New York, NY.

Ben-Arieh, D. and Chen, Z. (2006), "Linguistic labels aggregation and consensus measure for autocratic decision-making using group recommendations", IEEE Transactions on Systems, Man and Cybernetics, Part A: Systems and Humans, Vol. 36 No. 3, pp. 558-568.

Ben-Arieh, D. and Easton, T. (2007), "Multi-criteria group consensus under linear cost opinion elasticity", Decision support systems, Vol. 43 No. 3, pp. 713-721.

Ben-Arieh, D., Easton, T. and Evans, B. (2009), "Minimum cost consensus with quadratic cost functions", IEEE Transactions on Systems, Man and Cybernetics, Part A: Systems and Humans, Vol. 39 No. 1, pp. 210-217.

Bose, U., Davey, A.M. and Olson, D.L. (1997), "Multi-attribute utility methods in group decision making: Past applications and potential for inclusion in GDSS", Omega-International Journal of Management Science, Vol. 25 No. 6, pp. 691-706.

Brysont, N. (1996), "Group Decision-Making and the Analytic Hierarchy Process: Exploring the ConsensusRelevant Information Content", Computers $\&$ Operations Research, Vol. 23 No. 1, pp. 27-35. 
Brysont, N. (1997), "Supporting consensus formation in Group Support Systems using the Qualitative Discriminant Process", Annals of Operations Research, Vol. 71, pp. 75-91.

Bryson, N. and Joseph, A. (1999), "Generating consensus priority point vectors: a logarithmic goal programming approach", Computers \&3 Operations Research, Vol. 26 No. 6, pp. 637-643.

Cabrerizo, F.J., Moreno, J.M., Pérez, I.J. and Herrera-Viedma, E. (2010), "Analyzing consensus approaches in fuzzy group decision making: advantages and drawbacks", Soft Computing, Vol. 14 No. 5, pp. 451-463.

Chankong, V. and Haimes, Y.Y. (1983), Multiobjective Decision Making: Theory ang Methodology, Dover Publications, New York.

Chen, S.M. and Lee L.W. (2012), "Autocratic consensus decision making using group recommendations based on the ILLOWA Operator and likelihood-based comparison relations", IEEE Transaction on System, Man, and Cybernetics- Part A: System and Humans, Vol. 42 No. 1, pp. 115-129.

Chiclana, F., Herrera, F. and Herrera-Viedma, E. (2002), "A note on the internal consistency of various preference representations", Fuzzy Sets and Systems, Vol. 131 No. 1, pp. 75-78.

Eklund, P., Rusinowska, A. and Swart, H.D. (2007), "Consensus reaching in committees", European Journal of Operational Research, Vol. 178 No. 1, pp. 185-193.

Feng,Y., Xu, S. and Zhang, B. (2014), "Group consensus control for double-integrator dynamic multi-agent systems with fixed communication topology", International Journal of Robust and Nonlinear Control Vol. 24 No. 3, pp. 532-547.

Gong, Z.W., Zhang, H.H., Forrest, J., Li, L.S. and Xu X.X. (2015a), "Two consensus models based on the minimum cost and maximum return regarding either all individuals or one individual", European Journal of Operational Research Vol. 240 No. 1, pp. 183-192.

Gong, Z.W., Xu, X.X., Zhang, H.H., Ozturk, U.A., Herrera-viedma, E. and Xu, C. (2015b), "The Minimum Cost Consensus Models with Grey Preference Opinions and Their Economic Interpretation", OmegaInternational Journal of Management Science Vol. 55, pp. 81-90.

Gonzalez-Pachon, J. and Romero, C. (2011), "The design of socially optimal decisions in a consensus scenario", Omega-International Journal of Management Science, Vol. 39 No. 2, pp. 179-185.

Herrera, F., Herrera-Viedma, E. and Verdegay, J.L. (1996), "A model of consensus in group decision making under linguistic assessments", Fuzzy Sets and Systems, Vol. 78 No. 1, pp. 73-87.

Herrera, F., Martinez, L. and Sanchez, P.J. (2005), "Managing non-homogeneous information in group decision making", European Journal of Operational Research, Vol. 166 No. 1, pp. 115-132.

Herrera-Viedma, E., Alonso, S., Chiclana, F. and Herrera, F. (2007), "A consensus model for group decision making with incomplete fuzzy preference relations", IEEE Transactions on Fuzzy Systems, Vol. 15 No. 5, pp. 863-877. 
Herrera-Viedma, E., Cabrerizo, F.J., Kacprzyk, J. and Pedrycz, W. (2014), "A review of soft consensus models in a fuzzy environment", Information Fusion, Vol. 17, pp. 4-13.

Herrera-Viedma, E., Chiclana, F., Herrera, F. and Alonso, S. (2007), "Group decision-making model with incomplete fuzzy preference relations based on additive consistency systems", IEEE Transactions on Man, and Cybernetics, Part B: Cybernetics, Vol. 37 No. 1, pp. 176-189.

Herrera-Viedma, E., Martinez, L., Mata, F. and Chiclana, F. (2005), "A consensus support system model for group decision-making problems with multigranular linguistic preference relations", IEEE Transactions on Fuzzy Systems, Vol. 13 No. 5, pp. 644-658.

Houthakker, H.S. (1950), "Revealed preference and the utility function", Economica, Vol. 17 No. 66, pp. 159-174.

Lee, H.S. (2002), "Optimal consensus of fuzzy opinions under group decision making environment", Fuzzy Sets and Systems, Vol. 132 No. 3, pp. 303-315.

Levy, W.B. and Delic, H. (1994), "Maximum entropy aggregation of individual opinions", IEEE Transactions on Systems, Man and Cybernetics, Vol. 24 No. 4, pp. 606-613.

Linares, P. and Romero, C. (2002), "Aggregation of preferences in an environmental economics context: a goal programming approach", Omega-International Journal of Management Science, Vol. 30 No. 2, pp. 89-95.

Miao, G.Y. and Ma, Q. (2015), "Group consensus of the first-order multi-agent systems with nonlinear input constraints", Neurocomputing, Vol. 161, pp. 113-119.

Ness, J. and Hoffman, C. (1998), Putting Sense into Consensus: Solving the Puzzle of Making Team Decisions Tacoma, Vista Assoc, WA.

Pérez, I.J., Wikströ, R., Mezei, J., Carlsson, C. and Herrera-Viedma, E. (2013), "A new consensus model for group decision making using fuzzy ontology", Soft Computing, Vol. 17 No. 9, pp. 1617-1627.

Ramanathan, R. and Ramanathan, U. (2010), "A qualitative perspective to deriving weights from pairwise comparison matrices", Omega-International Journal of Management Science, Vol. 38 No. 3, pp. 228-232.

Saaty, T.L. and Rogers, P.C. (1976), "Higher education in the united states (1985-2000): Scenario construction using a hierarchical framework with eigenvector weighting", Socio-Economic Planning Sciences, Vol. 10 No. 6, pp. 251-263.

Shannon, C.E. (1948), "A mathematical theory of communication", Bell System Technical Journal, Vol. 27 No. 3, pp. 379-423.

Simon, H. (1991), "Bounded rationality and organizational learning", Organization Science, Vol. 2 No. 1, pp. 125-134. 




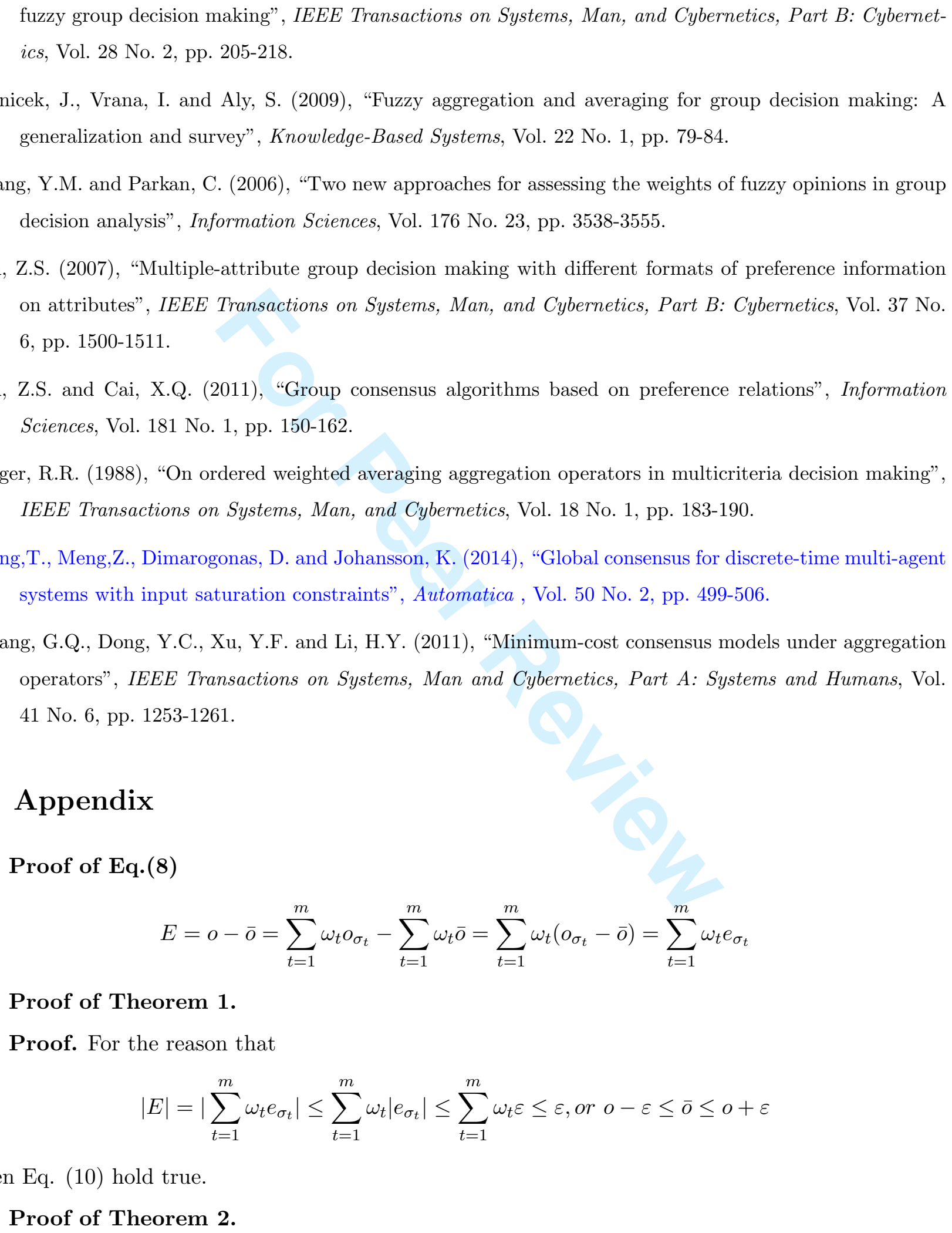

$$
E=o-\bar{o}=\sum_{t=1}^{m} \omega_{t} o_{\sigma_{t}}-\sum_{t=1}^{m} \omega_{t} \bar{o}=\sum_{t=1}^{m} \omega_{t}\left(o_{\sigma_{t}}-\bar{o}\right)=\sum_{t=1}^{m} \omega_{t} e_{\sigma_{t}}
$$

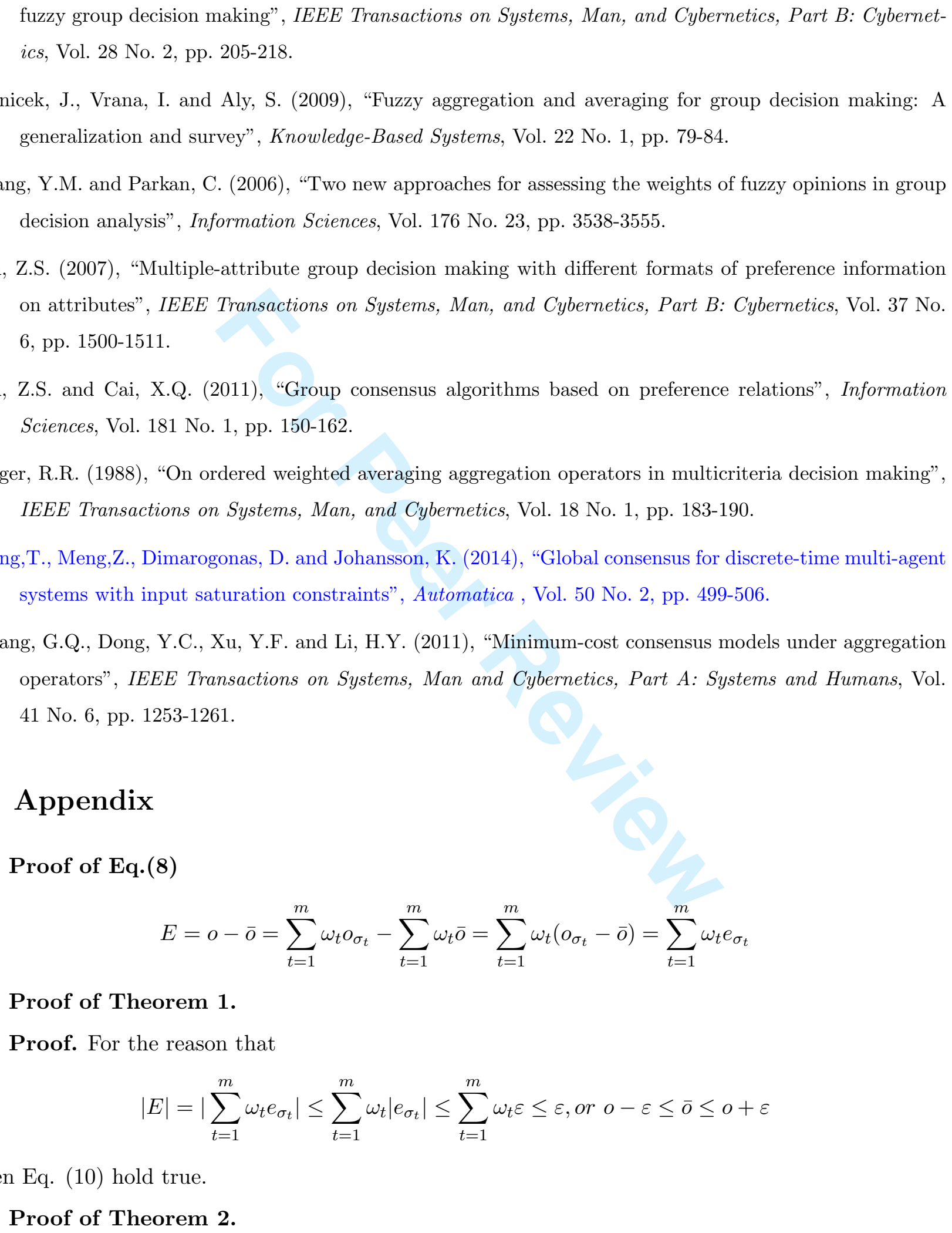

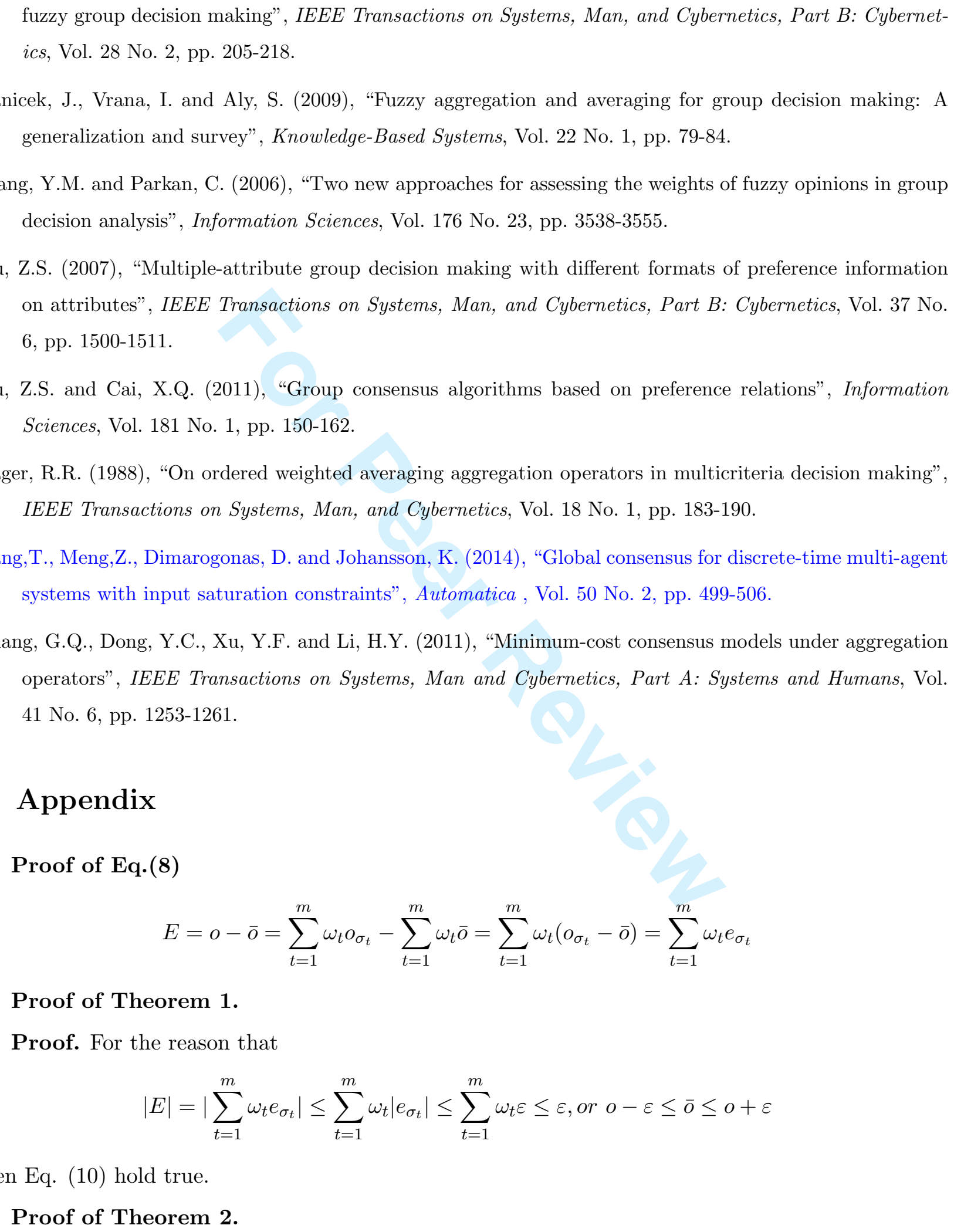

$$
|E|=\left|\sum_{t=1}^{m} \omega_{t} e_{\sigma_{t}}\right| \leq \sum_{t=1}^{m} \omega_{t}\left|e_{\sigma_{t}}\right| \leq \sum_{t=1}^{m} \omega_{t} \varepsilon \leq \varepsilon, \text { or } o-\varepsilon \leq \bar{o} \leq o+\varepsilon
$$

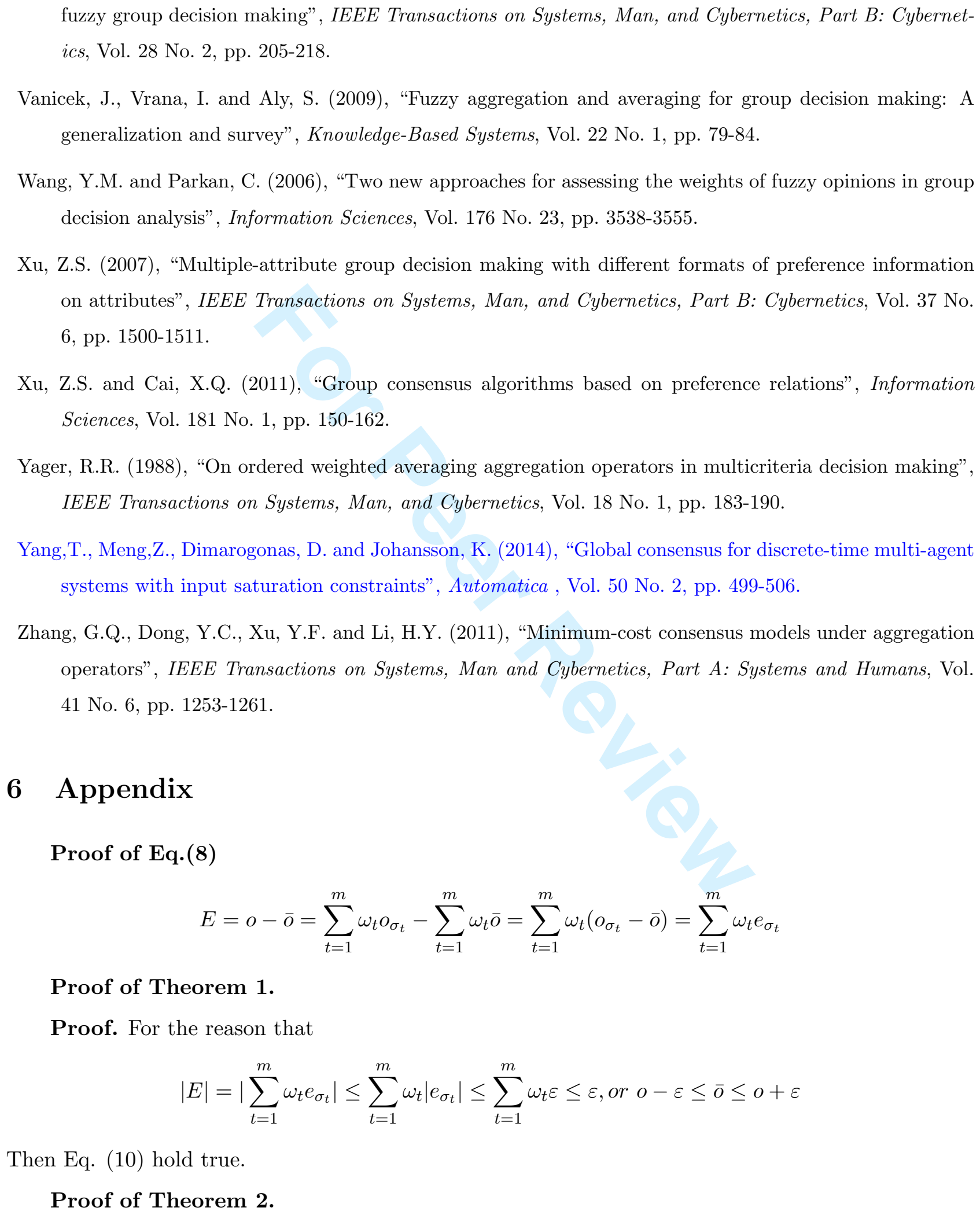

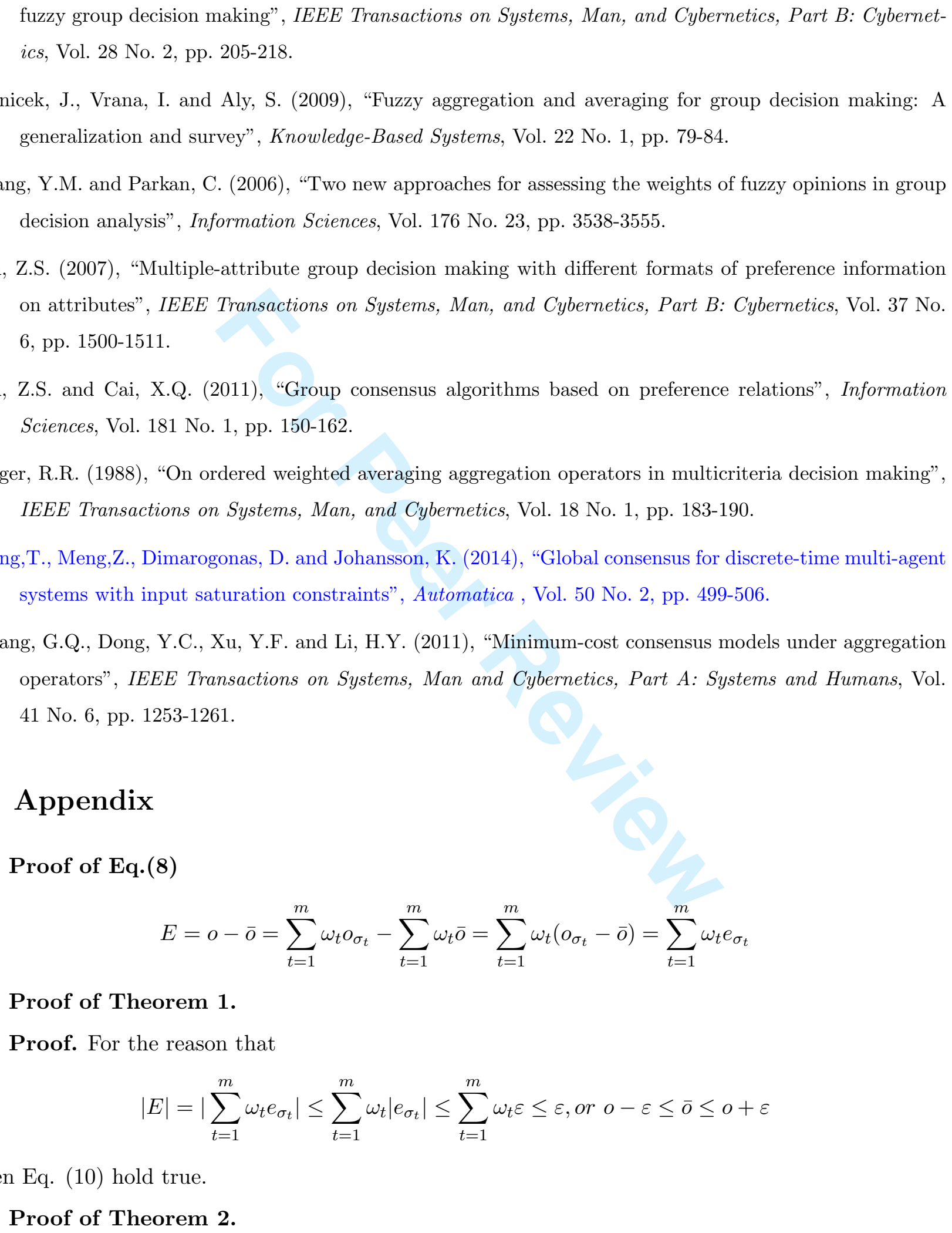

Proof. Let $\sum_{t=1}^{m} e_{t}=0$. Then $\sum_{t=1}^{m} \frac{1}{m} e_{t}=0$. That means that $\Omega=(1 / m, 1 / m, \ldots, 1 / m)^{T}$ is an optimal solution to the COWA Model, and $E(\Omega)=0$. 
Proof. It is obvious that $\Omega_{m}=\left(\begin{array}{lllll}0 & 0 & \ldots & 0 & 1\end{array}\right)^{T}$ is a feasible solution to the COWA Model. For any feasible solution $\Omega=\left(\omega_{1} \omega_{2} \ldots \omega_{m}\right)^{T}$ of the COWA Model satisfying $\sum_{t=1}^{m} \omega_{t}=1, \omega_{t} \geq 0$, we prove in the following that $E\left(\Omega_{m}\right) \leq E(\Omega)$. In particular, we have

$$
E\left(\Omega_{m}\right)=\left|e_{m}\right|=\left|\sum_{t=1}^{m} \omega_{t} e_{m}\right| \leq\left|\sum_{t=1}^{m} \omega_{t} e_{\sigma_{t}}\right|=E(\Omega)
$$

That is, $\min E(\Omega)=\left|e_{m}\right|$. Thus $\Omega_{m}=\left(\begin{array}{lllll}0 & 0 & \ldots & 0 & 1\end{array}\right)^{T}$ is an optimal solution to the COWA Model.

Proof of Theorem 4.

Proof. Suppose that there are $m$ DMs that participate in the consensus decision making, and that $\Omega^{*}=\left(\Omega_{1}^{*} \Omega_{2}^{*} \ldots \Omega_{m}^{*}\right)^{T}$ is an optimal solution to the COWA Model. Then we have

$$
E^{*}\left(\omega_{1}, \omega_{2}, \ldots, \omega_{m}\right)=\left|\sum_{t=1}^{m} \omega_{t}^{*} e_{\sigma_{t}}\right|
$$

where $\left\{\sigma_{1}, \sigma_{2}, \ldots, \sigma_{m}\right\}$ is a permutation of $\{1,2, \ldots, m\}$, and $e_{\sigma_{t-1}} \geq e_{\sigma_{t}}, t=2,3, \ldots, m ; \omega_{t}^{*}$ satisfying $\sum_{t=1}^{m} \omega_{t}^{*}=1, \omega_{t}^{*} \geq 0, t \in M$, are the importance of $d_{\sigma_{t}}$.

Now, let us add to the decision group $q, q \geq 1$, new DMs $d_{m+i}$ whose individuals' opinions are $o_{m+i}, i=1,2, \ldots, q$. Then the corresponding deviation is $e_{m+i}=o_{m+i}-\bar{o}, i=1,2, \ldots, q$. Then we have a new optimal weight vector $\bar{\Omega}^{*}=\left(\bar{\omega}_{1}^{*} \bar{\omega}_{2}^{*} \ldots \bar{\omega}_{m}^{*} \bar{\omega}_{m+1}^{*} \ldots \bar{\omega}_{m+q}^{*}\right)^{T}$ such that

$$
E^{*}\left(\omega_{1}, \omega_{2}, \ldots, \omega_{m}, \omega_{m+1}, \ldots, \omega_{m+q}\right)=\left|\sum_{t=1}^{m+q} \bar{\omega}_{t}^{*} e_{\bar{\sigma}_{t}}\right|
$$

where $\left\{\bar{\sigma}_{1}, \bar{\sigma}_{2}, \ldots, \bar{\sigma}_{m+q}\right\}$ is a permutation of $\{1,2, \ldots, m+q\}$, and $e_{\bar{\sigma}_{t-1}} \geq e_{\bar{\sigma}_{t}}, t=2,3, \ldots, m+q ; \omega_{t}^{*}$ satisfying where $\sum_{t=1}^{m+q} \bar{\omega}_{t}^{*}=1, \bar{\omega}_{t}^{*} \geq 0, t=1,2, \ldots, m+q$, are the importance of $d_{\bar{\sigma}_{t}}$.

Suppose that $\left\{e_{\bar{\sigma}_{1}}, e_{\bar{\sigma}_{1}}, \ldots, e_{\bar{\sigma}_{m+q}}\right\}=\left\{e_{\sigma_{1}}, \ldots, e_{\sigma_{m_{1}}^{\prime}}, e_{\sigma_{m_{1}}}, \ldots, e_{\sigma_{m_{2}}^{\prime}}, e_{\sigma_{m_{2}}}, \ldots, e_{\sigma_{m_{q}}^{\prime}}, e_{\sigma_{m_{q}}}, \ldots, e_{\sigma_{m}}\right\}$ satisfying $\left\{e_{\sigma_{1}} \geq \ldots \geq e_{\sigma_{m_{1}}^{\prime}} \geq e_{\sigma_{m_{1}}} \geq \ldots \geq e_{\sigma_{m_{2}}^{\prime}} \geq e_{\sigma_{m_{2}}} \geq \ldots e_{\sigma_{m_{q}}^{\prime}} \geq e_{\sigma_{m_{q}}} \geq \ldots \geq e_{\sigma_{m}}\right\}$ is a permutation of $\left\{e_{1}, e_{2}, \ldots, e_{m+q}\right\}$, and $\left\{e_{\sigma_{m_{1}}^{\prime}}, e_{\sigma_{m_{2}}^{\prime}}, \ldots, e_{\sigma_{m_{q}}^{\prime}}\right\}$ satisfying $e_{\sigma_{m_{1}}^{\prime}} \geq e_{\sigma_{m_{2}}^{\prime}} \geq \ldots \geq e_{\sigma_{m_{q}}^{\prime}}$ is a permutation of $\left\{e_{m+1}, e_{m+2}, \ldots, e_{m+q}\right\}$. Obviously, $L=\left(\omega_{1}^{*}, \omega_{2}^{*}, \ldots, \omega_{m_{1}-1}^{*}, 0, \omega_{m_{1}}^{*}, \ldots, \omega_{m_{2}-1}^{*}\right.$, $\left.0, \omega_{m_{2}}^{*}, \ldots, \omega_{m_{q}-1}^{*}, 0, \omega_{m_{q}}^{*}, \ldots, \omega_{m}^{*}\right)^{T}$ is a feasible solution to the COWA Model. So, we have

$$
\begin{aligned}
E^{*}\left(\omega_{1}, \omega_{2}, \ldots, \omega_{m+q}\right) & \leq E\left(\omega_{1}^{*}, \omega_{2}^{*}, \ldots, \omega_{m_{1}-1}^{*}, 0, \omega_{m_{1}}^{*}, \ldots, \omega_{m_{q}-1}^{*}, 0, \omega_{m_{q}}^{*}, \ldots, \omega_{m}^{*}\right)^{T} \\
& =\sum_{t=1}^{m_{1}-1} \omega_{t}^{*} e_{\sigma_{t}}+0 e_{\sigma^{\prime} m_{1}}+\sum_{t=m_{1}}^{m_{2}-1} \omega_{t}^{*} e_{\sigma_{t}}+\cdots+0 e_{\sigma^{\prime} m_{q}}+\sum_{t=m_{q}}^{m} \omega_{t}^{*} e_{\sigma_{t}} \\
& =\sum_{t=1}^{m} \omega_{t}^{*} e_{\sigma_{t}}+0 e_{\sigma^{\prime} m_{1}}+\cdots+0 e_{\sigma^{\prime} m_{q}} \\
& =E\left(\omega_{1}^{*}, \omega_{2}^{*}, \ldots, \omega_{m}^{*}\right) \\
& =E^{*}\left(\omega_{1}, \omega_{2}, \ldots, \omega_{m}\right)
\end{aligned}
$$


and

\section{Proof of Corollary $1 \mathrm{a}$ and Corollary $1 \mathrm{~b}$.}

Proof. Suppose the consensus threshold value is $\varepsilon, \varepsilon \geq 0$, and the consensus level based on the COWA model with $m$ DMs is acceptable. Then according to Theorem 4, we have

$$
E^{*}\left(\omega_{1}, \omega_{2}, \ldots, \omega_{m}, \ldots, \omega_{m+q}\right) \leq E^{*}\left(\omega_{1}, \omega_{2}, \ldots, \omega_{m}\right) \leq \sum_{i=1}^{m} \omega_{i}^{*}\left|e_{\sigma_{i}}\right| \leq \varepsilon
$$

$$
\sum_{i=1}^{m+q} \omega_{i}^{*}\left|e_{\sigma_{i}}\right| \leq \varepsilon
$$

This means that the GDM with $m+1$ DMs based on the COWA model is at a level of acceptable consensus. Therefore, both Corollary 1a and Corollary 1b hold true.

\section{Proof of Theorem 5.}

Proof. In the light of Theorem 3, when the sum of the individuals' deviations of the $m$ DMs is 0 , all the DMs have the same weight and the weight of each DM is $\frac{1}{m}$. The entropy of the GDM system is $F(m)=-\sum_{t=1}^{m} \omega_{t} \log \left(\omega_{t}\right)=-\sum_{t=1}^{m} \frac{1}{m} \log \left(\frac{1}{m}\right)=\log (m)$. Obviously, the inequality $F(m) \leq F(m+1)$ shows that the entropy of the GDM system increases with the number of DMs involved in the decision process. This completes the proof. 